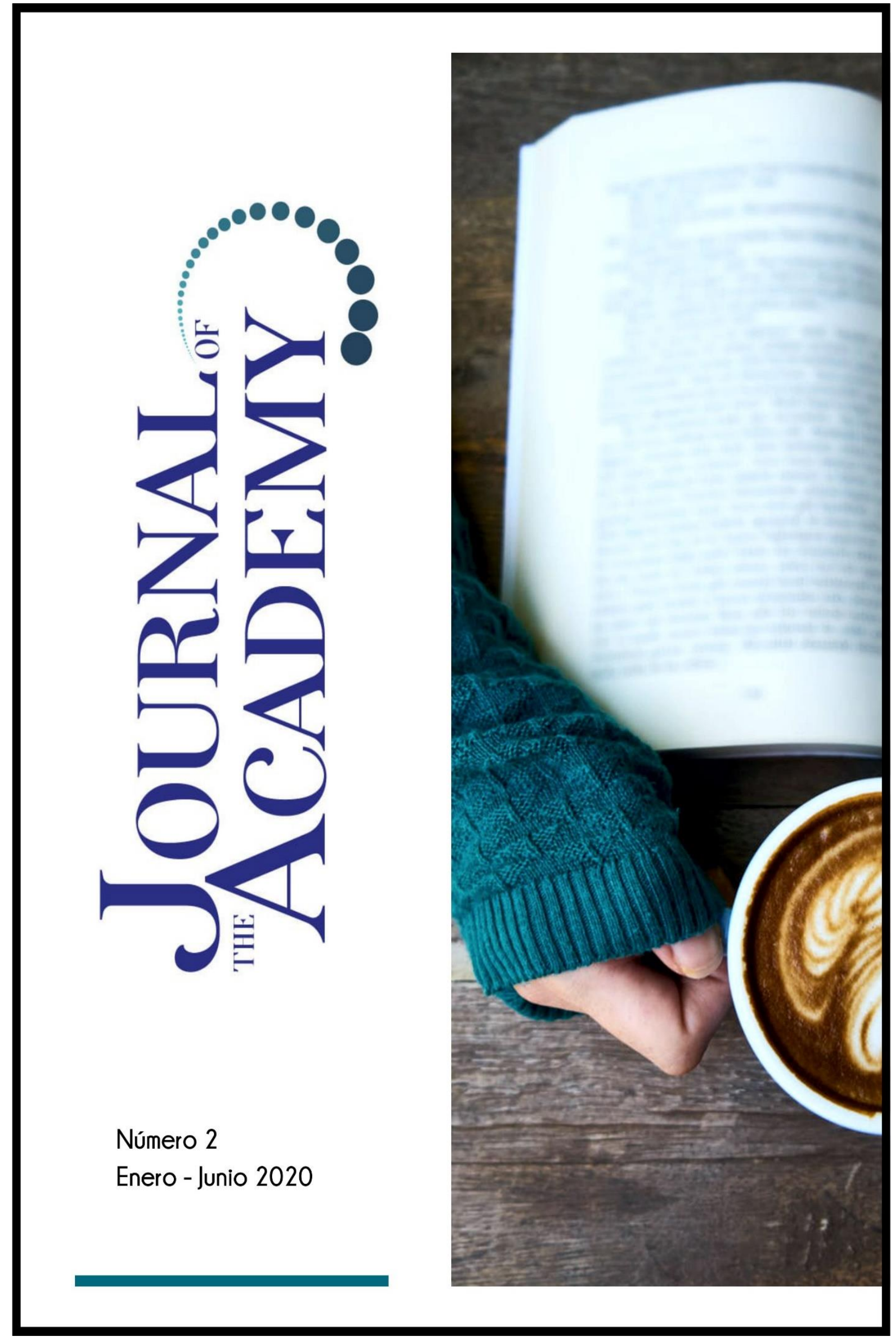




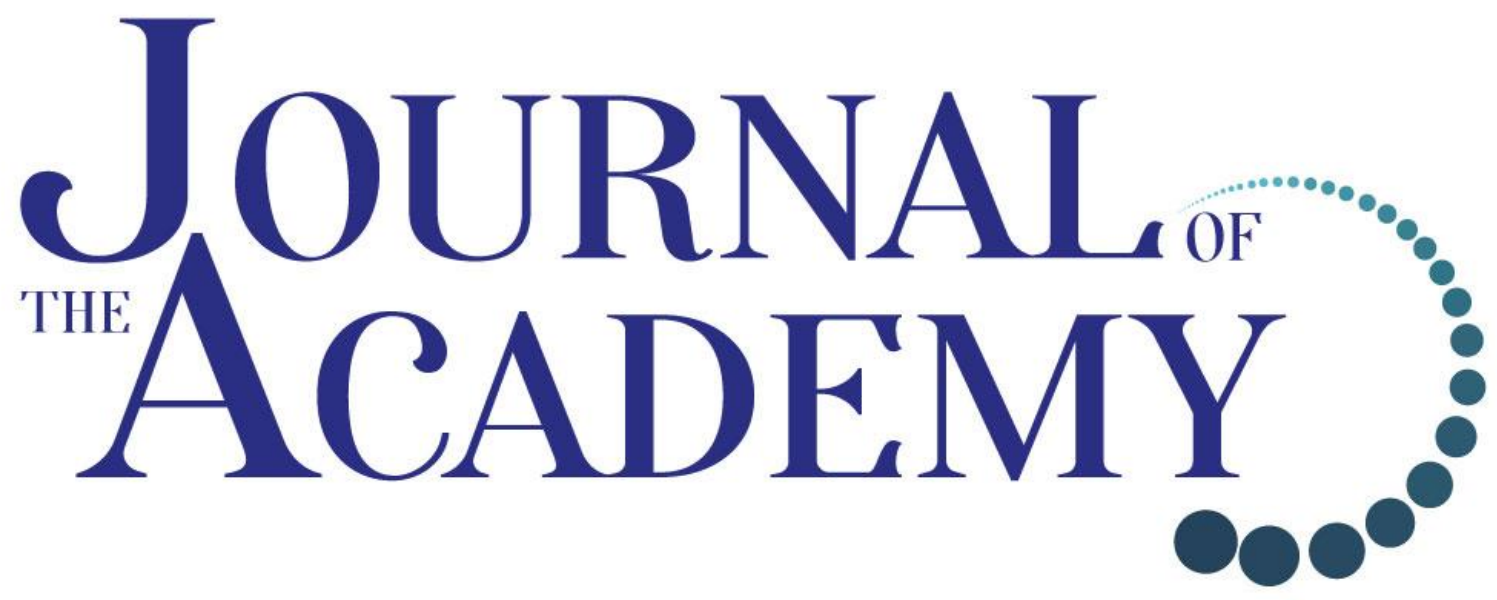

Publicación Científica de la Asociación de Universidades del Perú ASUP

Número 2

Enero-Junio 2020

www.journalacademy.org 


\section{ISSN 2707-0301}

\section{Directora-Editora en Jefe}

Ada Gallego Ruiz Conejo

Editor Científico

Oswaldo Orellana Manrique

Comité Científico

\section{Javier Carreón Guillén}

Universidad Nacional Autónoma de México, México Martino Contu

Universidad de Sassari, Italia

Jorge Enrique Elías Caro

Universidad del Magdalena, Colombia

Roberto Escalante Semerena

Universidad Nacional Autónoma de México, México

Oscar Ortega Arango

Universidad Autónoma de Yucatán, México

Alex Veliz Burgos

Universidad de Los Lagos, Chile

Comité Editorial

\section{Manuela Garau}

Centro Studi Sea, Italia

José Manuel González Freire

Universidad de Colima, México

Carlos Tulio da Silva Medeiros

Diálogos en Mercosur, Brasil

Eduardo Gomes Onofre

Universidade Estadual da Paraíba, Brasil

\section{Equipo Ejecutivo}

Juan Carlos Norabuena Castañeda

Thalia Chávez Cortéz

Journal Academy

Revista Semestral Open Access 


\title{
NIVEL DE LAS INTELIGENCIAS MÚLTIPLES DE ESTUDIANTES DEL 1RO Y 2DO DE SECUNDARIA EN LAS INSTITUCIONES EDUCATIVAS, PROVINCIA DE ANDAHUAYLAS, PERÚ, 2017
}

\section{LEVEL OF MULTIPLE INTELLIGENCES OF STUDENTS OF THE 1ST AND 2ND HIGH SCHOOL IN THE EDUCATIONAL INSTITUTIONS, PROVINCE OF ANDAHUAYLAS, PERU, 2017}

Recibido: 02 de junio de 2019

Aceptado: 15 de noviembre de 2019

\author{
Felipe Rafael VALLE díAZ \\ Universidad Nacional José María Arguedas, Perú \\ William Camilo YAURIS POLO \\ Universidad Nacional José María Arguedas, Perú \\ wyauris@gmail.com \\ Yudy PÉREZ ZÚÑIGA \\ Universidad Nacional José María Arguedas, Perú \\ wyauris@gmail.com
}

\begin{abstract}
The objective of the research was to determine the level of multiple intelligences in the students of the 1st and 2nd Secondary Education Institutions with Full and Regular School Day (1 stage) of the province of Andahuaylas. The population was students of 1 and 2 years of secondary school of six Secondary Schools, located in the valley Chumbao, province of Andahuaylas. The type of research is basic. The research design is descriptive-comparative.
\end{abstract}

Keywords: multiple intelligences, students, level

Resumen: El objetivo de la investigación fue determinar el nivel de las inteligencias múltiples en los estudiantes del 1 ro y 2 do de Secundaria de las Instituciones Educativas con Jornada Escolar Completa y Regular (1 etapa) de la provincia de Andahuaylas. La población fueron estudiantes del 1 y 2 año de secundaria de seis Colegios Secundarios, ubicados en el valle Chumbao, provincia de Andahuaylas. El tipo de investigación es básica. El diseño de investigación es descriptiva-comparativa.

Palabras claves: inteligencias múltiples, estudiantes, nivel

\section{Para Citar este Artículo:}

Valle Díaz, Felipe Rafael; Yauris Polo, William Camilo y Pérez Zúñiga, Yudy. Nivel de las inteligencias múltiples de estudiantes del 1ro y 2do de secundaria en las instituciones educativas, provincia de Andahuaylas, Perú, 2017. Journal of Academy num 1 (2019): 33-45.

Licencia Creative Commons Atributtion Nom-Comercial 3.0 Uunported

(CC BY-NC 3.0)

Licencia Internacional

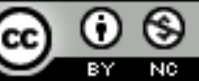




\section{Introducción}

"La palabra "inteligencia" tiene su origen en la unión de dos vocablos latinos: inter= entre, y eligere= escoger" (Antunes, 2000. Pág. 9). De esa capacidad cerebral que el ser humano posees y que lo desarrolla durante el transcurso de su vida y por otra parte, es muy importante el reconocer nuestras inteligencias y combinarlas de manera inteligente ya que ellas nos darán una mejor oportunidad para desenvolvernos de manera adecuada a los problemas que se nos presenten. (Gardner, 2006)

Howard Gardner a partir de 1983, sustenta la Teoría (IM) como un modelo de funcionamiento cognitivo que agrupa diferentes capacidades específicas, que poseen las personas, las cuales trabajan en forma conjunta determinando que existe muchas maneras de ser inteligentes, a pesar de estar genéticamente determinadas, pueden desarrollarse y mejorarse a través de la práctica y el aprendizaje, alcanzando cada categoría niveles adecuados de competencia dando lugar a un conjunto de inteligencias múltiples, distintas, diferenciadas entre sí e independientes pero interrelacionadas.

Actualmente los programas educativos que implementa el sistema peruano no contemplan ni mencionan directamente a las inteligencias múltiples como tal en los esquemas y contenidos programáticos a pesar que el Ministerio de Educación Peruano (MINEDU: 2017) establece en el diseño curricular qué " El propósito número 10 de la Educación Básica Regular del 2021" dice : "Desarrollo de la creatividad, innovación, apreciación y expresión a través de las artes, las humanidades y las ciencias", lo cual queda a merced de los profesores que elaboran su planificación didáctica, sin contemplar el beneficio que se puede lograr en cada estudiante indistintamente, quedando relegada a la iniciativa de cada docente apoyar y explorar las diversas facetas en el desarrollo de las inteligencias mediante la inclusión de acciones meditadas encaminadas a lograr una formación adecuada.

(Gadner: 1998) El Test de Inteligencias Múltiples valora a través de preguntas por ítems, clasificadas para las 8 inteligencias que miden el nivel de cada una de ellas, nos dan un resultado que nos sirve para establecer el diagnostico de las competencias del conocimiento, las aptitudes cognoscitivas básicas, las capacidades de expresión, las habilidades interpersonales, indicadores que nos sirven para orientar la futura vocación de los estudiantes, evitar frustraciones en sus estudios y afianzar la actividades prácticas cotidianas, que van a permitir a los estudiantes lograr la resolución de problemas y poder integrarse al ritmo con el que se desarrollan otras dimensiones de la vida social, es decir, aprender a emprender siendo uno de los objetivos esenciales del trabajo educativo.

El problema se evidenció a partir de resultados respecto a las capacidades lectoras relacionadas a recuperar información, inferir el significado del texto y reflexionar sobre la forma, el contenido y el contexto del texto, en los estudiantes del segundo año de secundaria a nivel de la provincia de Andahuaylas (MINEDU: 2017) presentan serios problemas porque no pueden inferir, reflexionar sobre la forma y contenido del texto expuesto, y sobre todo no recuperan información, aquí están contenidos más de los dos tercios de estudiantes censados, Estos problemas relacionados a las inteligencias lingüística, interpersonal, intrapersonal. Además, presentan problemas en aprendizajes respecto a determinar cantidad; regularidad, equivalencia y cambio; gestión de datos e incertidumbre; y forma, movimiento y localización relacionados a las inteligencias naturalista y corporal. Problemas fuertes en matematizar, razonar y argumentar, comunicar y representar; así como al elaborar y usar estrategias relacionados a las inteligencias matemática, espacial. (MINEDU, 2017). Los estudiantes del primero y segundo año de secundaria a nivel de la provincia de Andahuaylas, presentan serios problemas. 
Estadísticas reportadas del MINEDU, nos demuestra que solo el $1.4 \%$ de la población en edad escolar de secundaria, tiene un rendimiento suficiente en el área de matemática. Este indicador es regular, respecto a otras regiones con similar situación de pobreza. Esto significa que al terminar la secundaria, alrededor del 99\% no tendrá un rendimiento suficiente en el área de matemáticas.

Considerando la aplicación de los principios psicológicos en la práctica educativa, y teniendo como directrices las propiedades del aprendizaje se presenta el proyecto de investigación que permitirá brindar información clasificada para iniciar cambios cognoscitivos, emocionales en los estudiantes, que en sus cortas carreras escolares ya han acumulado muchos fracasos, teniendo en cuenta que las misma no ofrece trabajar desde un paradigma de crecimiento y de comprensión, sino desde el déficit como estamos acostumbrados en forma rutinaria y tradicional, obviando las particularidades de aprendizaje de cada estudiante. Por lo que se plantea la siguiente pregunta de la investigación:

¿Cuál es el nivel de las inteligencias múltiples en los estudiantes del 1 ro y 2 do de Secundaria de las Instituciones Educativas con Jornada Escolar Completa y Regular de la provincia de Andahuaylas, 20172019?

Determinar el nivel de las inteligencias múltiples en los estudiantes del 1ro y 2 do de Secundaria de las Instituciones Educativas con Jornada Escolar Completa y Regular de la provincia de Andahuaylas, 20172019.

La pertinencia se centra: la Universidad Nacional José María Arguedas, se ciñe a los artículos de la Ley 30220, para intervenir como promotor, responsable. Asimismo, está contenida dentro de las funciones del Ministerio de Educación a través de la Superintendencia Nacional de Educación Superior. Asimismo, existe convenio con la Unidad de Gestión Educativa Local Andahuaylas, lo cual fortalece la coordinación, integración de personal docente, administrativo de las instituciones educativas secundarias, que participan activamente en el proyecto. Esta investigación se ubicó en la línea de investigación Código CTI 02 Social sub área 02.01 Educación, en la línea específica 02.01.00.02 Aprendizajes pertinentes y de calidad.

La viabilidad se pone de manifiesto: se cuenta con recursos humanos suficientes para el soporte, seguimiento, por parte de la Universidad Nacional José María Arguedas, caso: Estadístico, economista, educador. Las instituciones educativas cuentan con especialistas es pedagogía, psicología, tutoría. El antecedente de soporte es aquel que se aplicó a ciento treinta y tres estudiantes de tercer grado de secundaria de una institución educativa de Ventanilla - Callao en el año 2010. El instrumento aplicado es la escala MINDS (mentes) de Inteligencias Múltiples, validado en el Perú por Ruiz (2004). El resultado indica niveles altos en las inteligencias Kinestésica, interpersonal y naturalista así como niveles bajos en las inteligencias matemáticas y lingüística del grupo de estudiantes. La aplicación del instrumento contribuye a la descripción de las inteligencias múltiples y la agrupación de los estudiantes por patrones de inteligencias por niveles altos, medios y bajos.

El segundo antecedente de soporte es Graciela de los Ángeles y Alarcón (2005). Las inteligencias múltiples en la escuela secundaria: caso de una institución pública del estado de México. Resultados: el concepto de inteligencia de alumnos y docentes dista del propuesto en la Teoría de Inteligencias múltiples. Es decir que existe poca intervención entre lo propuesto y lo realizado. Además, pone énfasis en que el currículo formal de manera implícita y fragmentaria propone el desarrollo del perfil intelectual de los adolescentes y deja a los profesores la responsabilidad discrecional de este desarrollo, lo que ellos abordan deficientemente. Llegó a la conclusión que es importante replantear la concepción de inteligencia 
dominante entre docentes y alumnos porque de ésta dependen en buena medida los objetivos y las estrategias educativas realmente asumidas por ellos. Es necesario reorientar el currículo formal y la formación magisterial para promover la estimulación integral del potencial intelectual entre los escolares. El modelo educativo debe centrarse en el aprendizaje, no en la enseñanza. En síntesis, Gardner (1998) plantea que:

- Las inteligencias múltiples son un medio para comprender el intelecto.

- Su fin es mejorar el entendimiento del mundo, es decir, comprender el mundo físico y el de la expresión humana.

- Una finalidad específica es la capacidad de usar el conocimiento en situaciones reales.

- La escuela brinda diferentes procesos y múltiples patrones de pensamiento.

- Para evaluar el entendimiento se requiere una evaluación auténtica.

- Está basada en el desempeño de los alumnos donde demuestran todas sus potencialidades en todas las áreas especialmente la cognición.

- Se atiende y respeta las diferencias individuales de los educandos.

\section{Metodología}

El tipo de investigación a utilizar es básica: Hernández (2010. p 157). El diseño de investigación es Descriptiva-comparativa: se realiza un estudio a una sola variable. La investigación realizada responde a un tipo no experimental. Hernández (2010 p. 205), definen la investigación no experimental como "la investigación que se realiza sin manipular deliberadamente variables. Población y muestra de 1830 estudiantes de seis Instituciones Educativas Secundarias. El propósito de esta investigación es caracterizar un fenómeno o situación e indicar sus rasgos más resaltantes y diferenciadores. Se buscó organizar, analizar, derivando conclusiones significativas según el test validado (Hernández, 2010, p.200). Las conclusiones se basaron en comparaciones, contrastes. El instrumento aplicado fue el test Escala MINDSIM probado por César Ruíz Trujillo (2004). Además de ficha de observación para registrar información o datos sobre las variables que tiene en mente (Hernández, 2010, p.200). La recolección de los datos se lleva a cabo al utilizar procedimientos estandarizados y aceptados por una comunidad científica. Para que una investigación sea creíble y aceptada por otros investigadores, debe demostrarse que se siguieron tales procedimientos. Como en este enfoque se pretende medir, los fenómenos estudiados deben poder observarse o referirse en el "mundo real". (Hernández 2010, p. 5).

El procedimiento fue el siguiente:

- Primero: Recojo de datos en base al test de medición de la inteligencia múltiple.

- Segundo: Calificación del test mediante los baremos.

- Tercero: Programación de variable en el software.

- Cuarto: Vaciado de datos hacia el software. Solo vaciamos los datos aplicado el baremo, desde el test evaluado ya se tiene cuál de las dos inteligencias múltiples, está en mayor desarrollo. Evaluación por niveles de percentil.

- Quinto: Integración de la información, depurar los valores perdidos. 
- Sexto: Obtenemos tabla de frecuencia y gráficos por sección, grado, colegio.

- Séptimo: Tabla de estadísticos descriptivos en base a los baremos del test aplicado MINDSIM.

- Octavo: Interpretamos los resultados.

- Noveno: Organizamos la información final.

- Décimo: Presentamos la información final depurada.

Para la sistematización y operación de datos nos apoyamos en el paquete estadístico SPSS 22.

\section{Resultados}

Los resultados por cada Institución Educativa Secundario de la Provincia de Andahuaylas con su respectivo procesamiento estadístico e interpretación de los resultados. se presentan a continuación.

\section{Nivel de Inteligencia Lógico-Matemática del $1^{\circ}$ y $2^{\circ}$ grado de secundaria de los seis Colegios}

Gráfico Nº7 Nivel de inteligencias múltiple Lógico-Matemático en las IE Manuel Vivanco Altamirano, Federico Villareal, Virgen del Carmen, Próceres de la Independencia Americana, Agropecuario $\mathrm{N}^{\circ} 08$, Gregorio Martinelly, provincia Andahuaylas, 2017.

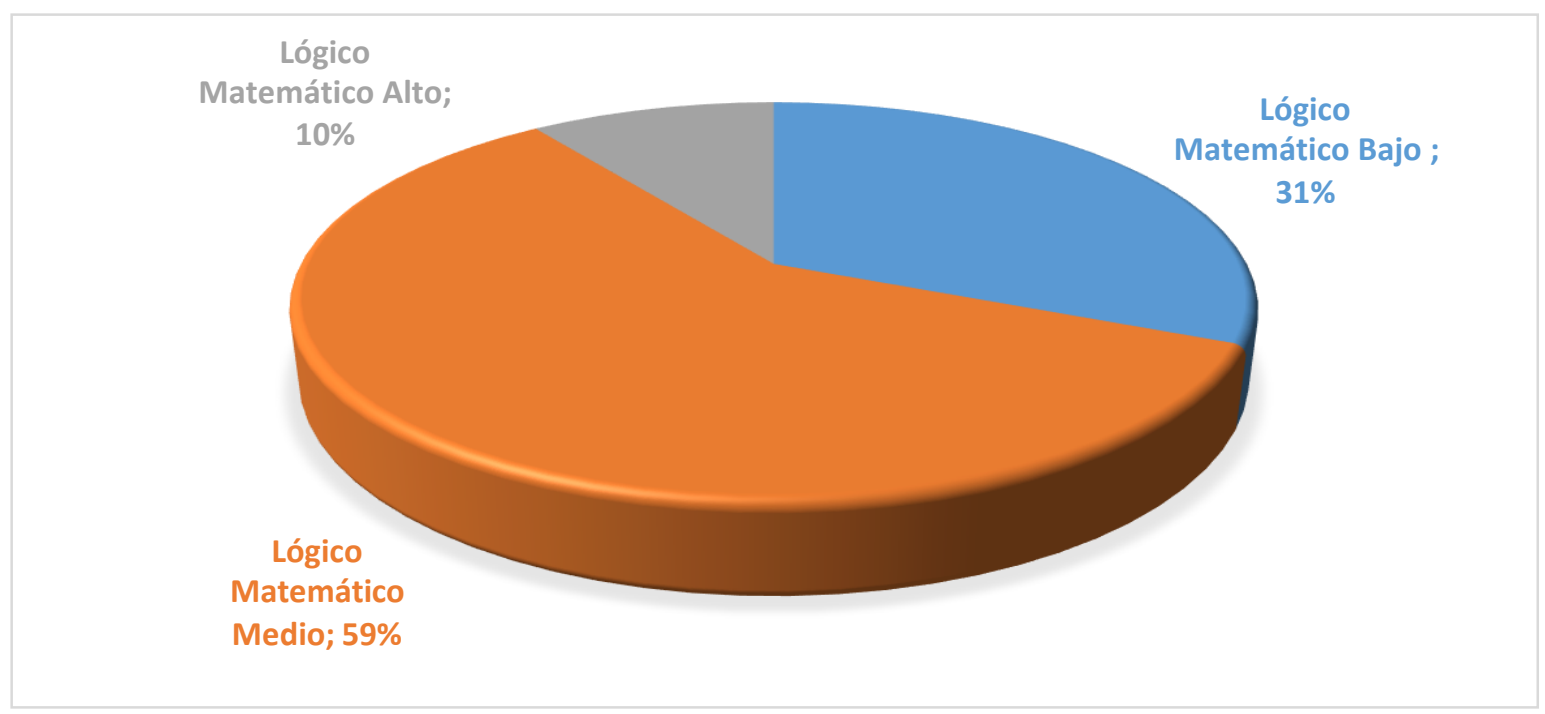

El $31 \%$ de estudiantes del $1^{\circ}$ y $2^{\circ}$ año de secundaria presentan un nivel bajo en inteligencia lógicomatemática. Los estudiantes con marcada tendencia bajo en lectura de mapas, gráficos, dibujando, laberintos, puzzles, imaginando cosas, visualizando; piensa en diseñar, dibujar, construir, crear, soñar despierto, mirar dibujos; aprenden mejor trabajando con dibujos y colores, visualizando, usando su ojo mental, dibujando. El $59 \%$ de estudiantes del $1^{\circ}$ y $2^{\circ}$ año de secundaria presentan un nivel medio en inteligencia lógico-matemático en lectura de mapas, gráficos, dibujando, laberintos, puzzles, imaginando cosas, visualizando; piensa en diseñar, dibujar, construir, crear, soñar despierto, mirar dibujos; aprenden mejor trabajando con dibujos y colores, visualizando, usando su ojo mental, dibujando. El $10 \%$ de estudiantes del $1^{\circ}$ y $2^{\circ}$ año de secundaria presentan un nivel alto en inteligencia Lógico-Matemático con marcada tendencia destacan en lectura de mapas, gráficos, dibujando, laberintos, puzzles, imaginando cosas, visualizando; piensa en diseñar, dibujar, construir, crear, soñar despierto, mirar dibujos; aprenden mejor trabajando con dibujos y colores, visualizando, usando su ojo mental, dibujando. 


\section{Nivel de Inteligencia Verbal-Lingüística del $1^{\circ}$ y $2^{\circ}$ grado de secundaria de los seis Colegios}

Gráfico Nº7 Nivel de inteligencias múltiple Lógico-Matemático en las IE Manuel Vivanco Altamirano, Federico Villareal, Virgen del Carmen, Próceres de la Independencia Americana, Agropecuario $\mathrm{N}^{\circ} 08$, Gregorio Martinelly, provincia Andahuaylas, 2017.

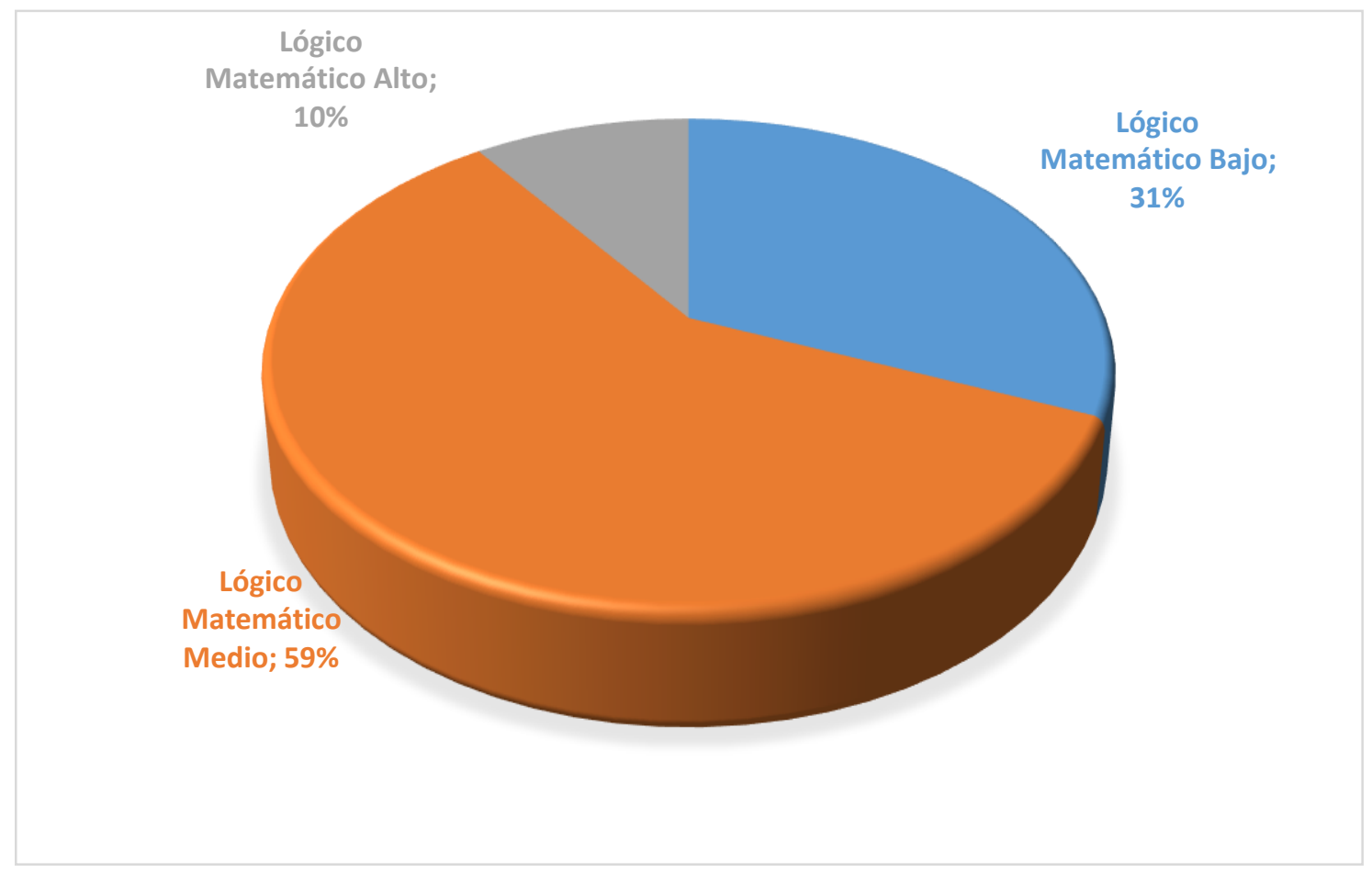

El $31 \%$ de estudiantes del $1^{\circ}$ y $2^{\circ}$ año de secundaria presentan un nivel bajo en inteligencia lógicomatemática. Los estudiantes con marcada tendencia bajo en lectura de mapas, gráficos, dibujando, laberintos, puzzles, imaginando cosas, visualizando; piensa en diseñar, dibujar, construir, crear, soñar despierto, mirar dibujos; aprenden mejor trabajando con dibujos y colores, visualizando, usando su ojo mental, dibujando. El $59 \%$ de estudiantes del $1^{\circ}$ y $2^{\circ}$ año de secundaria presentan un nivel medio en inteligencia lógico-matemático en lectura de mapas, gráficos, dibujando, laberintos, puzzles, imaginando cosas, visualizando; piensa en diseñar, dibujar, construir, crear, soñar despierto, mirar dibujos; aprenden mejor trabajando con dibujos y colores, visualizando, usando su ojo mental, dibujando. El 10\% de estudiantes del $1^{\circ}$ y $2^{\circ}$ año de secundaria presentan un nivel alto en inteligencia Lógico-Matemático con marcada tendencia destacan en lectura de mapas, gráficos, dibujando, laberintos, puzzles, imaginando cosas, visualizando; piensa en diseñar, dibujar, construir, crear, soñar despierto, mirar dibujos; aprenden mejor trabajando con dibujos y colores, visualizando, usando su ojo mental, dibujando.

\section{Nivel de Inteligencia Verbal-Lingüística del $1^{\circ}$ y $2^{\circ}$ grado de secundaria de los seis Colegios}

Gráfico $N^{\circ} 08$ Nivel de inteligencias múltiple Verbal-Lingüística en las IE Manuel Vivanco Altamirano, Federico Villareal, Virgen del Carmen, Próceres de la Independencia Americana, Agropecuario $\mathrm{N}^{\circ} 08$, Gregorio Martinelly, provincia Andahuaylas, 2017. 


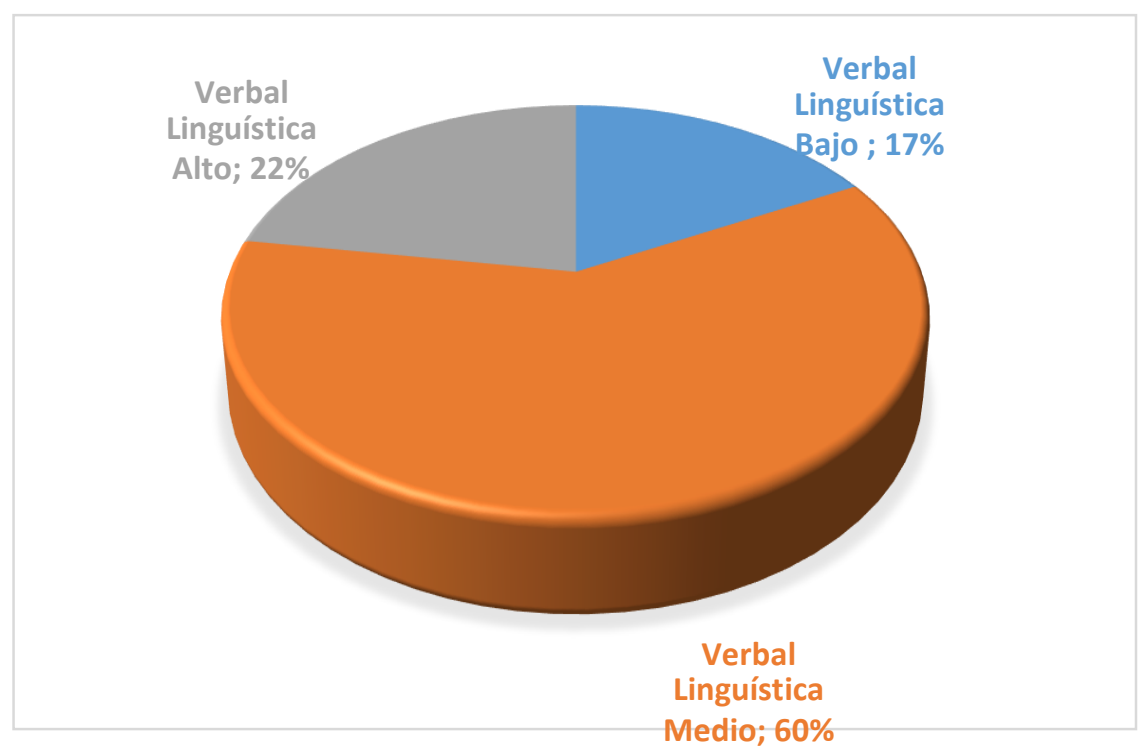

El $17 \%$ de estudiantes del $1^{\circ}$ y $2^{\circ}$ año de secundaria presentan un nivel bajo en inteligencia verballinguística. Los estudiantes con marcada tendencia bajo en lectura de mapas, gráficos, dibujando, laberintos, puzzles, imaginando cosas, visualizando; piensa en diseñar, dibujar, construir, crear, soñar despierto, mirar dibujos; aprenden mejor trabajando con dibujos y colores, visualizando, usando su ojo mental, dibujando. El $60 \%$ de estudiantes del $1^{\circ}$ y $2^{\circ}$ año de secundaria presentan un nivel medio en inteligencia verbal-lingüística en lectura de mapas, gráficos, dibujando, laberintos, puzzles, imaginando cosas, visualizando; piensa en diseñar, dibujar, construir, crear, soñar despierto, mirar dibujos; aprenden mejor trabajando con dibujos y colores, visualizando, usando su ojo mental, dibujando. El $10 \%$ de estudiantes del $1^{\circ}$ y $2^{\circ}$ año de secundaria presentan un nivel alto en inteligencia verbal-linguística con marcada tendencia destacan en lectura de mapas, gráficos, dibujando, laberintos, puzzles, imaginando cosas, visualizando; piensa en diseñar, dibujar, construir, crear, soñar despierto, mirar dibujos; aprenden mejor trabajando con dibujos y colores, visualizando, usando su ojo mental, dibujando.

\section{Nivel de Inteligencia Musical del $1^{\circ}$ y $2^{\circ}$ grado de secundaria de los seis Colegios}

Gráfico $N^{\circ} 09$ Nivel de inteligencias múltiple Musical en las IE Manuel Vivanco Altamirano, Federico Villareal, Virgen del Carmen, Próceres de la Independencia Americana, Agropecuario $N^{\circ}$ 08, Gregorio Martinelly, provincia Andahuaylas, 2017.

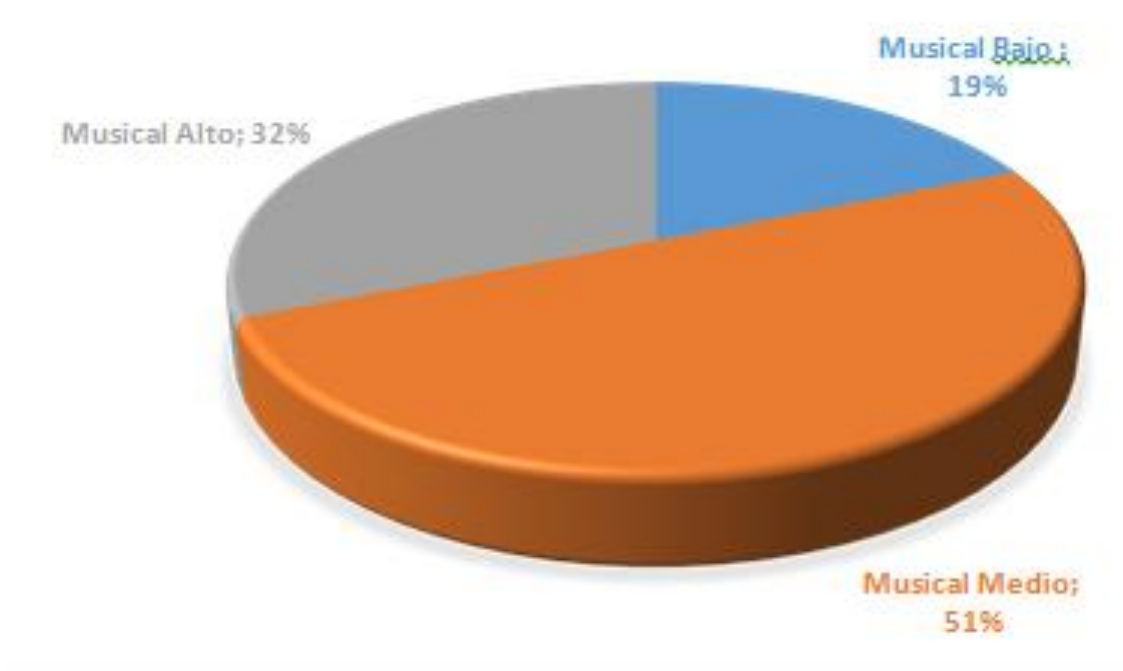


El $19 \%$ de estudiantes del $1^{\circ}$ y $2^{\circ}$ año de secundaria presentan un nivel bajo en inteligencia musical. Los estudiantes con marcada tendencia bajo en lectura de mapas, gráficos, dibujando, laberintos, puzzles, imaginando cosas, visualizando; piensa en diseñar, dibujar, construir, crear, soñar despierto, mirar dibujos; aprenden mejor trabajando con dibujos y colores, visualizando, usando su ojo mental, dibujando. El $51 \%$ de estudiantes del $1^{\circ}$ y $2^{\circ}$ año de secundaria presentan un nivel medio en inteligencia musical en lectura de mapas, gráficos, dibujando, laberintos, puzzles, imaginando cosas, visualizando; piensa en diseñar, dibujar, construir, crear, soñar despierto, mirar dibujos; aprenden mejor trabajando con dibujos y colores, visualizando, usando su ojo mental, dibujando. El $32 \%$ de estudiantes del $1^{\circ}$ y $2^{\circ}$ año de secundaria presentan un nivel alto en inteligencia musical con marcada tendencia destacan en lectura de mapas, gráficos, dibujando, laberintos, puzzles, imaginando cosas, visualizando; piensa en diseñar, dibujar, construir, crear, soñar despierto, mirar dibujos; aprenden mejor trabajando con dibujos y colores, visualizando, usando su ojo mental, dibujando.

\section{Nivel de Inteligencia Espacial del $1^{\circ}$ y $2^{\circ}$ grado de secundaria de los seis Colegios}

Gráfico $\mathrm{N}^{\circ} 10$ Nivel de inteligencias múltiple Espacial en las IE Manuel Vivanco Altamirano, Federico Villareal, Virgen del Carmen, Próceres de la Independencia Americana, Agropecuario $N^{\circ} 08$, Gregorio Martinelly, provincia Andahuaylas, 2017.

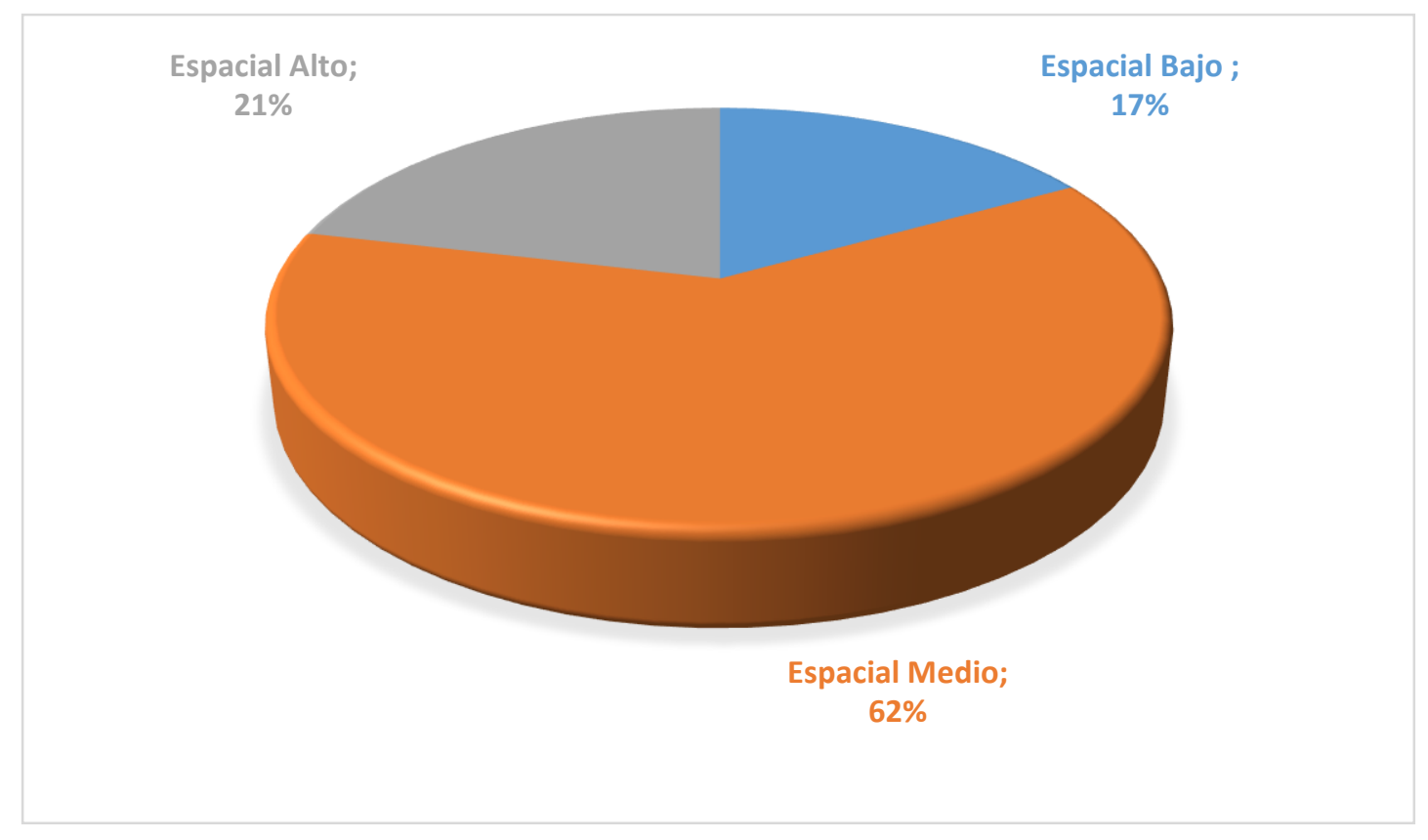

El $17 \%$ de estudiantes del $1^{\circ}$ y $2^{\circ}$ año de secundaria presentan un nivel bajo en inteligencia Espacial. Los estudiantes con marcada tendencia bajo en lectura de mapas, gráficos, dibujando, laberintos, puzzles, imaginando cosas, visualizando; piensa en diseñar, dibujar, construir, crear, soñar despierto, mirar dibujos; aprenden mejor trabajando con dibujos y colores, visualizando, usando su ojo mental, dibujando. El $62 \%$ de estudiantes del $1^{\circ}$ y $2^{\circ}$ año de secundaria presentan un nivel medio en inteligencia espacial en lectura de mapas, gráficos, dibujando, laberintos, puzzles, imaginando cosas, visualizando; piensa en diseñar, dibujar, construir, crear, soñar despierto, mirar dibujos; aprenden mejor trabajando con dibujos y colores, visualizando, usando su ojo mental, dibujando. El $21 \%$ de estudiantes del $1^{\circ}$ y $2^{\circ}$ año de secundaria presentan un nivel alto en inteligencia Espacial con marcada tendencia destacan en lectura 
de mapas, gráficos, dibujando, laberintos, puzzles, imaginando cosas, visualizando; piensa en diseñar, dibujar, construir, crear, soñar despierto, mirar dibujos; aprenden mejor trabajando con dibujos y colores, visualizando, usando su ojo mental, dibujando.

\section{Nivel de Inteligencia Intrapersonal del $1^{\circ}$ y $2^{\circ}$ grado de secundaria de los seis Colegios}

Gráfico $\mathrm{N}^{\circ} 12$ Nivel de inteligencias múltiple Intrapersonal en las IE Manuel Vivanco Altamirano, Federico Villareal, Virgen del Carmen, Próceres de la Independencia Americana, Agropecuario $\mathrm{N}^{\circ} 08$, Gregorio Martinelly, provincia Andahuaylas, 2017.

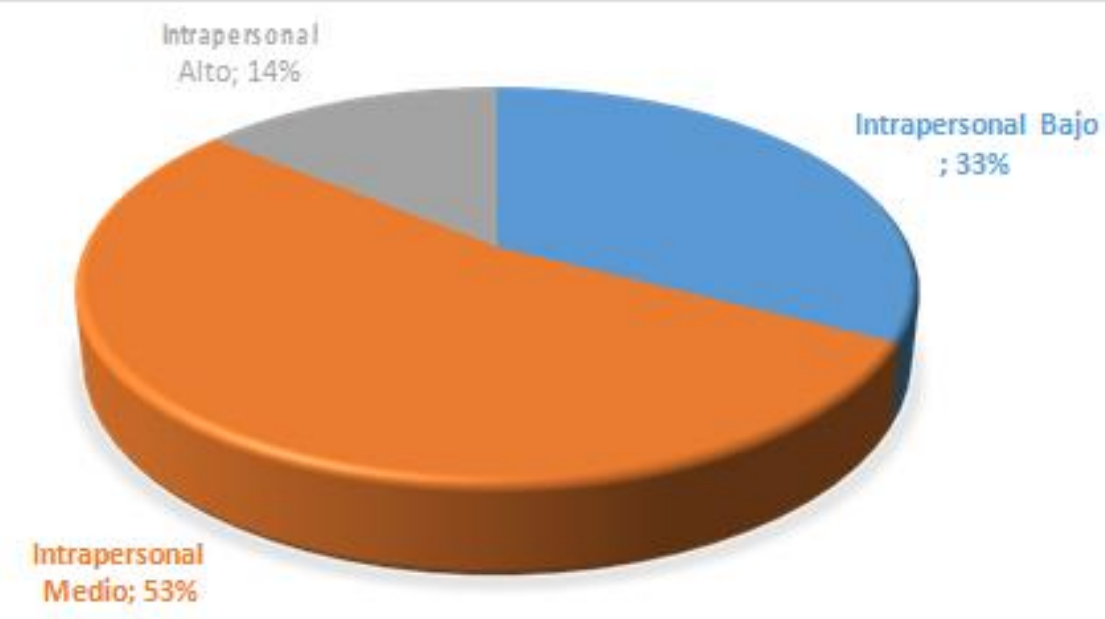

El $33 \%$ de estudiantes del $1^{\circ}$ y $2^{\circ}$ año de secundaria presentan un nivel bajo en inteligencia intrapersonal. Los estudiantes con marcada tendencia bajo en lectura de mapas, gráficos, dibujando, laberintos, puzzles, imaginando cosas, visualizando; piensa en diseñar, dibujar, construir, crear, soñar despierto, mirar dibujos; aprenden mejor trabajando con dibujos y colores, visualizando, usando su ojo mental, dibujando. El 53\% de estudiantes del $1^{\circ}$ y $2^{\circ}$ año de secundaria presentan un nivel medio en inteligencia intrapersonal en lectura de mapas, gráficos, dibujando, laberintos, puzzles, imaginando cosas, visualizando; piensa en diseñar, dibujar, construir, crear, soñar despierto, mirar dibujos; aprenden mejor trabajando con dibujos y colores, visualizando, usando su ojo mental, dibujando. El $14 \%$ de estudiantes del $1^{\circ}$ y $2^{\circ}$ año de secundaria presentan un nivel alto en inteligencia intrapersonal con marcada tendencia destacan en lectura de mapas, gráficos, dibujando, laberintos, puzzles, imaginando cosas, visualizando; piensa en diseñar, dibujar, construir, crear, soñar despierto, mirar dibujos; aprenden mejor trabajando con dibujos y colores, visualizando, usando su ojo mental, dibujando. 


\section{Nivel de Inteligencia Interpersonal del $1^{\circ}$ y $2^{\circ}$ grado de secundaria de los seis Colegios}

Gráfico $N^{\circ} 13$ Nivel de inteligencias múltiple Interpersonal en las IE Manuel Vivanco Altamirano, Federico Villareal, Virgen del Carmen, Próceres de la Independencia Americana, Agropecuario $\mathrm{N}^{\circ}$ 08, Gregorio Martinelly, provincia Andahuaylas, 2017

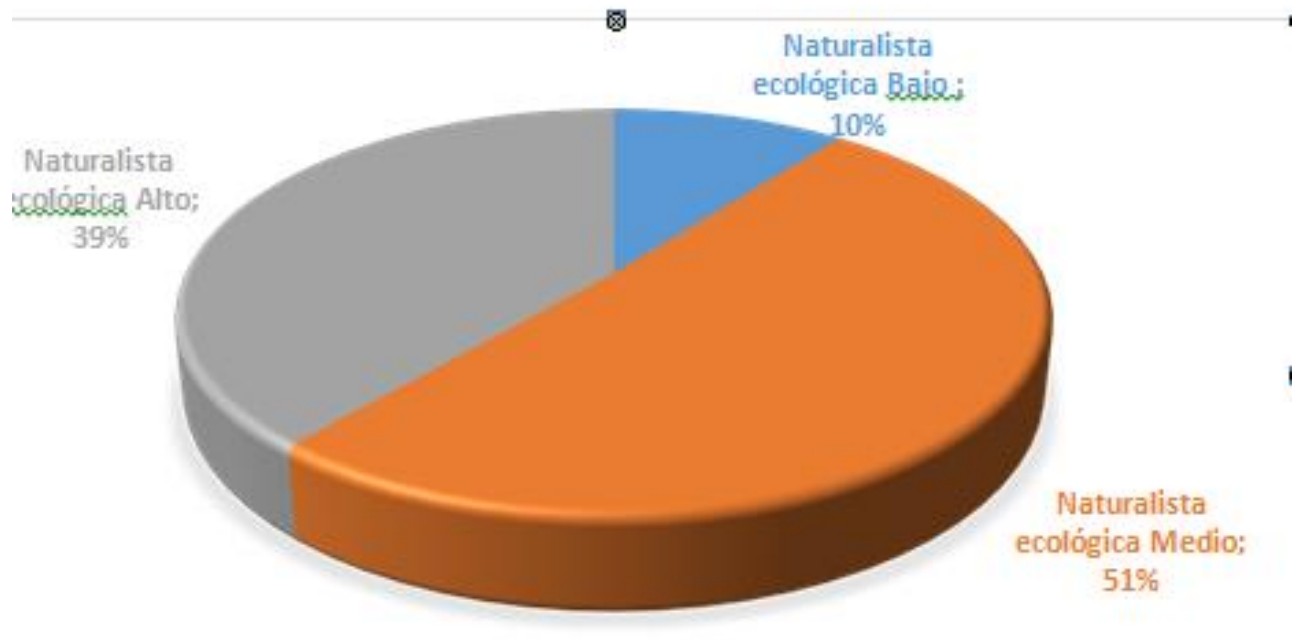

El $10 \%$ de estudiantes del $1^{\circ}$ y $2^{\circ}$ año de secundaria presentan un nivel bajo en inteligencia corporalnaturalista. Los estudiantes con marcada tendencia bajo en lectura de mapas, gráficos, dibujando, laberintos, puzzles, imaginando cosas, visualizando; piensa en diseñar, dibujar, construir, crear, soñar despierto, mirar dibujos; aprenden mejor trabajando con dibujos y colores, visualizando, usando su ojo mental, dibujando. El $51 \%$ de estudiantes del $1^{\circ}$ y $2^{\circ}$ año de secundaria presentan un nivel medio en inteligencia corporal-naturalista, en lectura de mapas, gráficos, dibujando, laberintos, puzzles, imaginando cosas, visualizando; piensa en diseñar, dibujar, construir, crear, soñar despierto, mirar dibujos; aprenden mejor trabajando con dibujos y colores, visualizando, usando su ojo mental, dibujando. El $39 \%$ de estudiantes del $1^{\circ}$ y $2^{\circ}$ año de secundaria presentan un nivel alto en inteligencia corporalnaturalista, con marcada tendencia destacan en lectura de mapas, gráficos, dibujando, laberintos, puzzles, imaginando cosas, visualizando; piensa en diseñar, dibujar, construir, crear, soñar despierto, mirar dibujos; aprenden mejor trabajando con dibujos y colores, visualizando, usando su ojo mental, dibujando.

\section{Discusión de resultados}

Según Howard Gardner, cada persona no tiene un sólo tipo de inteligencia sino ocho o nueve diferentes y además, cada persona posee mediante demostraciones prácticas o a través de 'inteligencias' al conjunto de habilidades, talentos capacidades mentales necesarias para resolver problemas o para elaborar productos que son de importancia en un contexto cultural o en una comunidad determinada. Presenta los resultados del objetivo general y un breve resumen de los resultados específicos. 


\begin{tabular}{|c|c|c|c|c|c|c|c|c|c|c|c|c|c|c|c|c|c|c|c|c|c|c|c|c|}
\hline & \multicolumn{3}{|c|}{ Verbal Linguistica final } & \multicolumn{3}{|c|}{ Lógico Matemático final } & \multicolumn{3}{|c|}{ Musical final } & \multicolumn{3}{|c|}{ Espacial final } & \multicolumn{3}{|c|}{ Corporal Kinestésica final } & \multicolumn{3}{|c|}{ Interpersonal final } & \multicolumn{3}{|c|}{ Intrapersonal final } & \multicolumn{3}{|c|}{ Naturalista ecológica final } \\
\hline & Bajo & Medio & Alto & Bajo & Medio & Alto & Ваjo & Medio & & Bajo & Medio & Alto & Ваjo & Medio & Alto & Bajo & Medio & Alto & Bajo & Medio & Alto & Bajo & Medio & Alto \\
\hline $\begin{array}{l}\text { Manuel Vivanco } \\
\text { Altamirano }\end{array}$ & $17 \%$ & $54 \%$ & $28 \%$ & $33 \%$ & $57 \%$ & $10 \%$ & $20 \%$ & $50 \%$ & $30 \%$ & $14 \%$ & $62 \%$ & $24 \%$ & $14 \%$ & $49 \%$ & $38 \%$ & $29 \%$ & $55 \%$ & $16 \%$ & $19 \%$ & $61 \%$ & $20 \%$ & $11 \%$ & $47 \%$ & $42 \%$ \\
\hline Federico Villareal & $18 \%$ & $61 \%$ & $22 \%$ & $34 \%$ & $54 \%$ & $12 \%$ & $12 \%$ & $63 \%$ & $25 \%$ & $18 \%$ & $64 \%$ & $18 \%$ & $7 \%$ & $52 \%$ & $42 \%$ & $27 \%$ & $61 \%$ & $12 \%$ & $100 \%$ & $0 \%$ & $0 \%$ & $9 \%$ & $52 \%$ & $39 \%$ \\
\hline Virgen del Carmen & $9 \%$ & $61 \%$ & $30 \%$ & $29 \%$ & $62 \%$ & $9 \%$ & $19 \%$ & $54 \%$ & $27 \%$ & $12 \%$ & $62 \%$ & $25 \%$ & $8 \%$ & $50 \%$ & $43 \%$ & $22 \%$ & $63 \%$ & $15 \%$ & $12 \%$ & $65 \%$ & $23 \%$ & $6 \%$ & $43 \%$ & $51 \%$ \\
\hline $\begin{array}{l}\text { Próceres dela } \\
\text { independencia }\end{array}$ & $21 \%$ & $60 \%$ & $20 \%$ & $30 \%$ & $59 \%$ & $11 \%$ & $24 \%$ & $50 \%$ & $27 \%$ & $21 \%$ & $56 \%$ & $23 \%$ & $14 \%$ & $44 \%$ & $42 \%$ & $22 \%$ & $62 \%$ & $17 \%$ & $22 \%$ & $62 \%$ & $16 \%$ & $15 \%$ & $55 \%$ & $30 \%$ \\
\hline Agropecuario N'08 & $17 \%$ & $63 \%$ & $21 \%$ & $29 \%$ & $61 \%$ & $11 \%$ & $20 \%$ & $25 \%$ & $61 \%$ & $16 \%$ & $62 \%$ & $21 \%$ & $10 \%$ & $54 \%$ & $36 \%$ & $28 \%$ & $60 \%$ & $12 \%$ & $20 \%$ & $67 \%$ & $12 \%$ & $12 \%$ & $54 \%$ & $33 \%$ \\
\hline Gregorio Martinelly & $22 \%$ & $64 \%$ & $14 \%$ & $34 \%$ & $59 \%$ & $7 \%$ & $19 \%$ & $61 \%$ & $20 \%$ & $19 \%$ & $66 \%$ & $16 \%$ & $12 \%$ & $51 \%$ & $37 \%$ & $24 \%$ & $66 \%$ & $10 \%$ & $22 \%$ & $65 \%$ & $13 \%$ & $9 \%$ & $54 \%$ & $37 \%$ \\
\hline
\end{tabular}

El nivel de inteligencia verbal-lingüística general para las seis instituciones educativas, presenta los siguientes valores; el nivel bajo alcanza el $17 \%$, el nivel medio alcanza el $60 \%$ y el nivel alto $23 \%$. El nivel medio es el mayor, frecuente e incidente. Lo cual da a conocer que estos estudiantes aprenden mejor, destaca y les gusta en estas áreas que a continuación presentamos según la tabla de Gardner:

\begin{tabular}{|c|c|c|c|c|}
\hline AREA & & DESTACA EN & LE GUSTA & APRENDE MEJOR \\
\hline $\begin{array}{l}\text { AREA } \\
\text { VERBAL }\end{array}$ & LINGÜÍSTICO- & $\begin{array}{lr}\text { Lectura, escritura, } \\
\text { narración de historias, } \\
\text { memorización de } \\
\text { fechas, piensa en } \\
\text { palabras }\end{array}$ & $\begin{array}{l}\text { Leer, escribir, } \\
\text { contar cuentos, } \\
\text { hablar, } \\
\text { memorizar, hacer } \\
\text { puzzles }\end{array}$ & $\begin{array}{l}\text { Leyendo, escuchando y } \\
\text { viendo palabras, } \\
\text { hablando, escribiendo, } \\
\text { discutiendo y debatiendo }\end{array}$ \\
\hline
\end{tabular}

En esta área lingüística los estudiantes se desenvuelven mejor según los resultados que se obtuvo con la investigación por otra parte según:

A Thurstone (1887-1955) se debe el desarrollo del análisis factorial en los EEUU. Su trabajo lo centró principalmente en la medida de las aptitudes intelectuales. Según él, el factor general "g", formulado anteriormente por Spearman, no describe con rigurosidad qué es la inteligencia; lo cual lo llevó a orientar sus investigaciones hacia análisis de los componentes de la inteligencia. Thurstone (1938) extrajo siete habilidades mentales primarias: comprensión verbal; fluidez verbal o rapidez lingüística capacidad para el cálculo; rapidez perceptiva; representación espacial; memoria y razonamiento inductivo. Para Thurstone, cualquier factor que exista ha de ser considerado como de segundo orden, según la correlación que tenga con las habilidades mentales primarias. Fundamentándose en esta teoría de las habilidades mentales, diseñó el test conocido como el PMA (Primary Mental Abilities Test), que se utilizó durante muchos años como un poderoso instrumento para la escolaridad y la orientación.

El nivel de inteligencia lógico-matemático general para las seis instituciones educativas, presenta los siguientes valores; el nivel bajo alcanza el $31 \%$, es te valor es preocupante por lo que esta área necesita mayor atención por cuanto a la enseñanza que se está brindando en los colegios, en el nivel medio alcanza el $59 \%$ y el nivel alto $10 \%$. El nivel medio es el mayor, frecuente e incidente. Además, el nivel bajo es preocupante supera el $25 \%$ del total. 
El nivel de inteligencia musical general para las seis instituciones educativas, presenta los siguientes valores; el nivel bajo alcanza el $19 \%$, el nivel medio alcanza el $51 \%$ y el nivel alto $32 \%$. El nivel medio es el mayor, frecuente e incidente. Además el nivel alto es muy preferente porque supera el $30 \%$ del total.

El nivel de inteligencia espacial general para las seis instituciones educativas, presenta los siguientes valores; el nivel bajo alcanza el $17 \%$, el nivel medio alcanza el $62 \%$ y el nivel alto $21 \%$. El nivel medio es el mayor, frecuente e incidente

El nivel de inteligencia corporal-kinestésica general para las seis instituciones educativas, presenta los siguientes valores; el nivel bajo alcanza el $11 \%$, el nivel medio alcanza el $50 \%$ y el nivel alto $39 \%$. El nivel medio es el mayor, frecuente e incidente. Además el nivel alto es muy preferente porque supera el $35 \%$ del total.

El nivel de inteligencia interpersonal general para las seis instituciones educativas, presenta los siguientes valores; el nivel bajo alcanza el $25 \%$, el nivel medio alcanza el $61 \%$ y el nivel alto $14 \%$. El nivel medio es el mayor, frecuente e incidente. Además el nivel bajo es preocupante supera el $25 \%$ del total.

El nivel de inteligencia intrapersonal general para las seis instituciones educativas, presenta los siguientes valores; el nivel bajo alcanza el 33\%, el nivel medio alcanza el 53\% y el nivel alto $14 \%$. El nivel medio es el mayor, frecuente e incidente. Además el nivel bajo es preocupante supera el $30 \%$ del total.

El nivel de inteligencia naturalista-ecológica general para las seis instituciones educativas, presenta los siguientes valores; el nivel bajo alcanza el $10 \%$, el nivel medio alcanza el $51 \%$ y el nivel alto $39 \%$. El nivel medio es el mayor, frecuente e incidente. Además el nivel alto es muy preferente porque supera el $35 \%$ del total.

Podemos comprobar que existen diferencias significativas, según los niveles alcanzados. Algunas inteligencias presentan niveles bajos fuertes, caso lógico- matemático, interpersonal e intrapersonal. Algunas inteligencias presentan niveles altos muy considerables, caso corporal- kinestésica, naturalistaecológica, musical.

\section{Conclusiones}

Los resultados arrojados nos indica que existen diferencias significativas en el nivel de las inteligencias múltiples en los estudiantes del 1ro y 2 do de Secundaria de las Instituciones Educativas con Jornada Escolar Completa y Regular (1 etapa) de la provincia de Andahuaylas, 2017-2019.

El nivel alto de cualquier inteligencia múltiple no llega al $25 \%$ del total de resultados. El nivel bajo en algunas inteligencias múltiples, que son lógico-matemático, intrapersonal, e interpersonal; supera el $25 \%$ del total de resultados. El nivel medio de las inteligencias múltiples es superior al $50 \%$ del total de resultados. Esto nos indica que existen diferencias significativas, que parten de problemas en lo interpersonal, intrapersonal y lógico-matemático. Además, que la diferencia significativa se centra en dos inteligencias múltiples, que son Corporal-Kinestésica, y Naturalista-Ecológica, donde el nivel bajo no supera el $11 \%$ del total de resultados y el nivel alto por encima del $35 \%$ del total de resultados.

Para los objetivos específicos, podemos precisar que existen diferencias significativas, porque los niveles de porcentaje son heterogéneos. Para todas las inteligencias múltiples el nivel bajo se ubica entre el 17\% al 33\% (excluyendo las inteligencias Corporal-Kinestésica y Naturalista-Ecológica), los niveles altos se 
ubica entre el $10 \%$ al $22 \%$; aquí excluimos la inteligencia musical). Un caso especial es la inteligencia musical, cuyo nivel bajo es $19 \%$ y el nivel alto es $32 \%$. Podemos precisar que una menor diferencia, determina el nivel medio para todas las inteligencias, cuyos valores son $50 \%$ mínimo al $62 \%$ máximo.

\section{Recomendaciones}

1. Proseguir con los estudios de inventario en las instituciones educativas, que no han sido intervenidas, para hallar el nivel de inteligencias múltiples por cada institución educativa, ubicadas en la provincia de Andahuaylas.

2. A partir de la Unidad Educativa de Gestión Local Andahuaylas, los especialistas deben coordinar e implementar una estrategia a partir de los resultados para fortalecer los puntos críticos, es decir las inteligencias múltiples débiles, a partir de las inteligencias fuertes. Luego implementar una estrategia para sostener e impulsar las inteligencias fuertes, que coadyuvan a las otras inteligencias.

3. A partir de las instituciones educativas secundarias evaluadas, se deben formar comités de trabajo que formulen las estrategias, programa de intervención con el acompañamiento de los especialistas y psicólogos.

\section{Referencias bibliográficas}

Antunes, C. (2000). Inteligencias Múltiples, Como Estimularlas y Desarrollarlas, Madrid: Narcea, S.A de Ediciones.

A Thurstone (1887-1955) Habilidades mentales primarias, desarrollo del análisis del factor general " $g$ ", EEUU.

Matos, F. M. (2012) Inteligencias múltiples en estudiantes de tercer grado de secundaria de una institución educativa de Ventanilla - Callao.

Gadner. H. (1998) Inteligencias múltiples: la teoría y la práctica. Barcelona: Editorial Paidós.

Gardner, H; Emma; Laskin, E. (2006). Mentes Líderes, Una Anatomía del liderazgo. Barcelona: PAIDOS Ibérica S.A.

De los Ángeles y Alarcón, G. (2005) Las inteligencias múltiples en la escuela secundaria: caso de una institución pública del estado de México.

Hernández Sampieri \& Collado \& Baptista. (2010) Metodología de la investigación. México: Ed. Pearson. 